\title{
Peptide Side-COOH Groups Have Two Distinct Conformations under Biorelevant Conditions
}

\author{
Oleksandr O. Sofronov, ${ }^{\text {IT }}$ Giulia Giubertoni, ${ }^{\text {TL }}$ Alberto Pérez de Alba Ortíz, Bernd Ensing, \\ and Huib J. Bakker*
}

Cite This: J. Phys. Chem. Lett. 2020, 11, 3466-3472

Read Online

ACCESS |

山ll Metrics \& More

回 Article Recommendations

Supporting Information

ABSTRACT: The carboxyl $(\mathrm{COOH})$ side chain groups of amino acids, such as aspartic acid, play an important role in biochemical processes, including enzymatic proton transport. In many theoretical studies, it was found that the (bio)chemical reactivity of the carboxyl group strongly depends on the conformation of this group. Interestingly, up to now there has been no experimental investigation of the geometry and the stability of different $\mathrm{COOH}$ conformers under biorelevant conditions. Here, we investigate the conformational isomerism of the side chain $\mathrm{COOH}$ group of $N$ acetyl aspartic acid amide using polarization-resolved two-dimensional infrared spectroscopy. We find that the carboxyl group shows

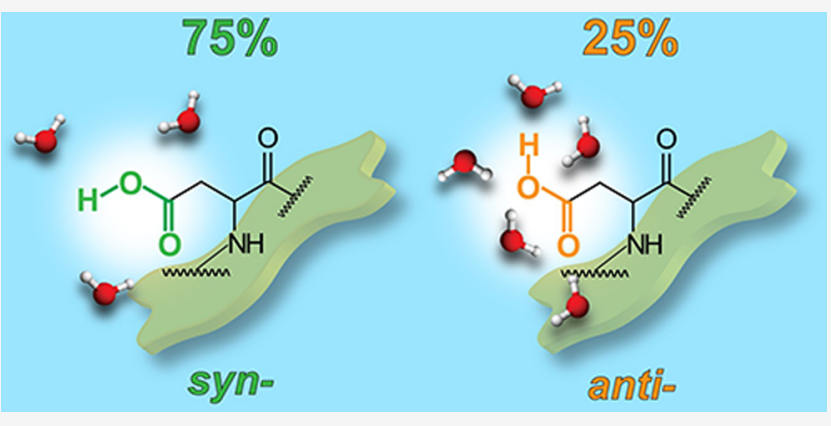
two distinct near-planar conformers (syn and anti) when dissolved in water at room temperature. Both conformers are significantly populated in aqueous solution $(75 \pm 10 \%$ and $25 \pm 10 \%$ for syn and anti, respectively). Molecular dynamics simulations show that the anti conformer interacts more strongly with water molecules than the syn conformer, explaining why this conformer is significantly present in aqueous solution.

C arboxyl groups and carboxylate anions are widely present in proteins, both as part of the side chain of amino acids glutamic and aspartic acid and as C-terminal groups. Under physiological conditions, the carboxyl groups are usually deprotonated, but in specific microenvironments, they can still be found in protonated form. ${ }^{1-4}$ The side chain carboxyl groups of aspartic acid and glutamic acid residues are essential for enzymatic catalysis, protein folding, ${ }^{5,6}$ proton conduction in protein nanochannels, ${ }^{7,8}$ and gating of $\mathrm{pH}$-sensing ion channels. ${ }^{9,10}$

For simple carboxylic acids (formic, acetic, and propionic acid) dissolved in rare-gas matrices under cryogenic conditions, the carboxyl group can adopt two distinctly different conformations with a planar structure. ${ }^{11-13}$ In the so-called syn conformer, the hydroxyl group is at an angle of $\sim 60^{\circ}$ with respect to the carbonyl group, while in the so-called anti conformer the hydroxyl group is oriented antiparallel to the carboxyl group. Recently, we showed for formic acid that distinct long-lived syn and anti carboxyl conformers exist also in aqueous solutions at room temperature, with relative abundances of $70-80 \%$ and $20-30 \%$, respectively. ${ }^{14}$

The conformational isomerism of carboxyl groups in molecules that are more complex than propionic acid has not been studied yet. ${ }^{15}$ The potential presence of the anti conformer is often neglected, especially in X-ray diffraction studies in which proton positions cannot be determined accurately. As a consequence, only $2 \%$ of the carboxyl groups found in the Cambridge Structural Database are depicted in an anti conformation. ${ }^{16}$ Nevertheless, the presence of side chain $\mathrm{COOH}$ groups of aspartic and glutamic acid residues in an anti conformation is expected to play an important role in enzymatic reactions and polypeptide structure stabilization. $^{16-23}$

Here we use polarization-resolved two-dimensional infrared (2DIR) spectroscopy and molecular dynamics simulations to study the conformational isomerism of the side chain carboxyl group of $\mathrm{N}$-acetyl aspartic acid amide (Figure 1), as a model for the aspartic acid residue. In the 2DIR experiments, we measure the response of the hydroxyl vibration upon the excitation of the carbonyl vibration of the same carboxyl group. The polarization dependence of this signal provides unique information on the molecular geometry of the carboxyl group. We observe two distinct conformations of the carboxyl group with different orientations of the hydroxyl group with respect to the carbonyl group. We also determine the fractions of the two conformers in dimethyl sulfoxide (DMSO) solution and in aqueous solution. Finally, we use molecular dynamics simulations to explain the structures and relative abundances

Received: March 4, 2020

Accepted: April 15, 2020

Published: April 15, 2020 


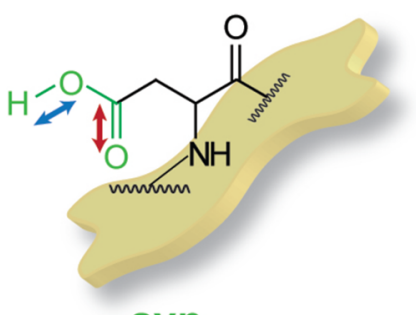

syn=

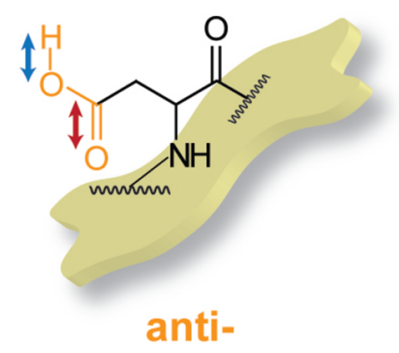

Figure 1. Syn and anti conformers of $\mathrm{N}$-acetyl aspartic acid amide. Red and blue arrows indicate the transition dipole moments of the carbonyl and hydroxyl vibrations, respectively.

of the two conformers from their intermolecular interactions with the solvent molecules.

First, we measure the 2DIR spectra of $N$-acetyl aspartic acid amide in DMSO solution $(0.4 \mathrm{M})$ obtained by exciting the carbonyl stretch vibration and probing the hydroxyl stretch vibration (Figure 2a). The good solubility of this amino acid in

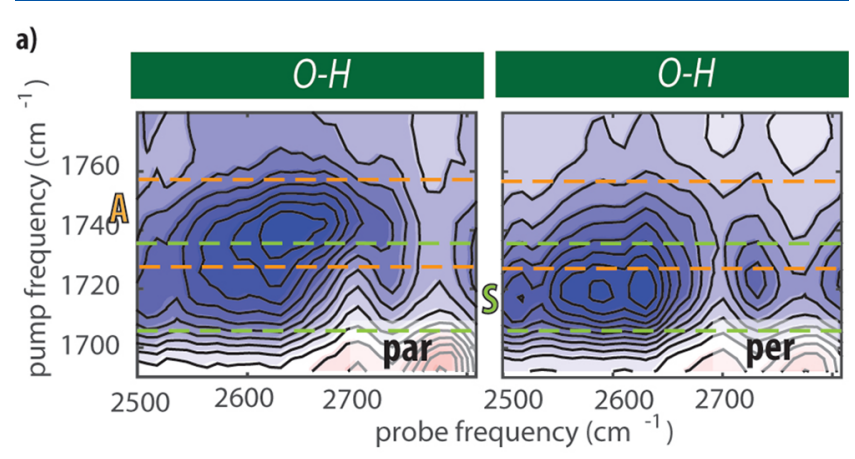

b)

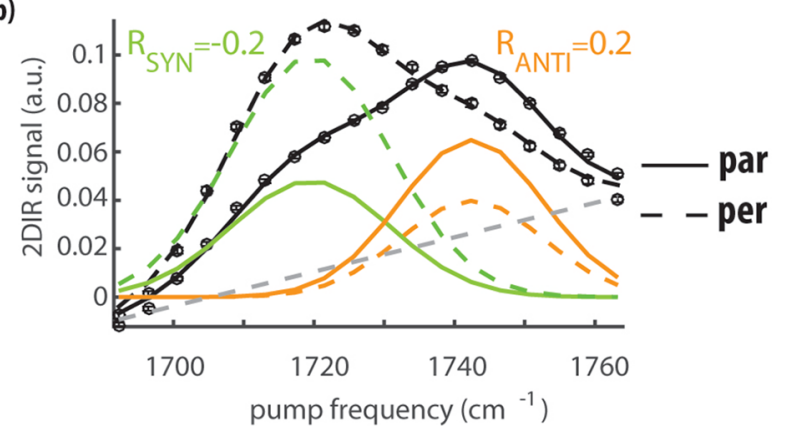

Figure 2. (a) 2DIR spectra of $\mathrm{N}$-acetyl aspartic acid amide in DMSO solution in the $\mathrm{C}=\mathrm{O} / \mathrm{O}-\mathrm{H}$ cross-peak region measured in parallel and perpendicular polarization configuration at a time delay $T_{\mathrm{w}}=0.5$ ps. The green and the orange bars highlight the responses of the syn and anti conformers, respectively. (b) Parallel (solid) and perpendicular (dashed) transient absorption signals obtained by integrating the corresponding $2 \mathrm{DIR}$ signal over the probe frequency in the range between 2500 and $2750 \mathrm{~cm}^{-1}$. The green and the orange colors indicate, respectively, the vibrational responses of the syn and anti conformers. The gray dashed line represents the correction for the isotropic background (see Methods section).

DMSO and the absence of solvent absorption bands in the spectral regions of the carbonyl and hydroxyl groups allow for an accurate measurement of the 2DIR cross-peak signals of these two groups. The carbonyl vibration has an absorption band centered at $1720-1730 \mathrm{~cm}^{-1}$. Upon excitation of this vibration, cross-peak signals appear at 2500-2800 $\mathrm{cm}^{-1}$ resulting from the excitation-induced frequency shift of the hydroxyl vibration. ${ }^{14}$ In the probed frequency region $2500-$
$2800 \mathrm{~cm}^{-1}$, this shift results in a negative absorption change (colored in blue). The cross-peak signal has the form of a series of subbands, which is typical for $\mathrm{OH}$-stretch vibrations of strongly hydrogen bonded systems, and results from the strong coupling of the $\mathrm{OH}$-stretch vibration to the low-frequency vibrations of the hydrogen bonds. ${ }^{24,25}$ These subbands are also observed in the linear infrared absorption spectrum of the hydroxyl vibration (Figure S1).

The amplitude of the cross-peak signal is observed to depend on the excitation frequency and the polarization configuration. Excitation of the low-frequency part of the carbonyl vibration $\left(\sim 1720 \mathrm{~cm}^{-1}\right)$ results in a stronger hydroxyl cross-peak signal in perpendicular polarization, whereas excitation of the high frequency part of the carbonyl peak $\left(\sim 1745 \mathrm{~cm}^{-1}\right)$ yields a cross-peak signal that is more pronounced in parallel polarization.

In Figure $2 b$, we show the 2DIR signals in parallel and perpendicular polarizations integrated over probe frequency range $2500-2750 \mathrm{~cm}^{-1}$ as a function of the carbonyl excitation frequency. We fit this integrated cross-peak signal with two Gaussian-shaped bands. For each band, we calculate the anisotropy, $R=\frac{\Delta \alpha_{\mathrm{par}}-\Delta \alpha_{\mathrm{per}}}{\Delta \alpha_{\mathrm{par}}+2 \Delta \alpha_{\mathrm{per}}}$, where $\Delta \alpha_{\mathrm{par}}$ and $\Delta \alpha_{\mathrm{per}}$ are the transient absorption changes (cross-peak signals) measured in parallel and in perpendicular polarization configuration, respectively. The anisotropy represents the relative orientation of the hydroxyl transition dipole moment with respect to the carbonyl transition dipole moment and can be used to calculate the angle between the carbonyl and the hydroxyl groups following $\theta=\arccos \sqrt{\frac{5 R_{0}+1}{3}}$.

We find that the cross-peak of the higher carbonyl vibration has a positive anisotropy of approximately $0.2\left(35^{\circ}\right)$ and the lower carbonyl peak has a negative anisotropy of approximately $-0.2\left(90^{\circ}\right)$. These anisotropy values clearly indicate that the two carboxyl group species comprise distinctly different relative orientations of the $\mathrm{O}-\mathrm{H}$ group and $\mathrm{C}=\mathrm{O}$ group. Based on the found angles, we assign the cross-peak signal with positive anisotropy and a high frequency of the carbonyl vibration to the anti conformer and the cross-peak signal with a negative anisotropy and a low frequency of the carbonyl vibration to the syn conformer. This result demonstrates that the carboxyl group shows distinct syn and anti conformers, not only for simple carboxylic acids but also for more complex molecules like amino acids. We observe these distinct conformers also for the terminal carboxyl groups of other amino acids in DMSO solution (Figures S2 and S3).

The obtained angles between the carbonyl and hydroxyl groups differ from the ideal values of $0^{\circ}$ (for a perfect in-plane anti parallel configuration) and $60^{\circ}$ (for an in-plane syn configuration). Similar deviations have been observed for formic acid in DMSO solution. ${ }^{14}$ These deviations can be explained from the fact that the extracted anisotropy values can be affected by out-of-plane rotations of the $\mathrm{O}-\mathrm{H}$ or $\mathrm{C}=\mathrm{O}$ group. Such a rotational distortion can explain an increase of the ideal angle of the anti configuration from $0^{\circ}$ to $35^{\circ}$ and an increase of the ideal angle of the syn configuration from $60^{\circ}$ to $\sim 90^{\circ}$. Analysis of the Cambrige Structural Database shows that the $\mathrm{O}-\mathrm{C}-\mathrm{O}-\mathrm{H}$ dihedral angle can deviate by $10-30^{\circ}$ from the ideal value for both the syn and anti conformers. ${ }^{26}$ In addition, the anisotropy values may be affected by fast inertial (librational) motion of the $\mathrm{O}-\mathrm{H}$ bond, which leads to an 
ultrafast or even pulse-width limited partial decrease of anisotropy. $^{27}$

To mimic the properties of aspartic acid residues under biological conditions, we study the conformational isomerism of $N$-acetyl aspartic acid amide in aqueous solution. In Figure 3 , we show 2DIR spectra of $\mathrm{N}$-acetyl aspartic acid amide

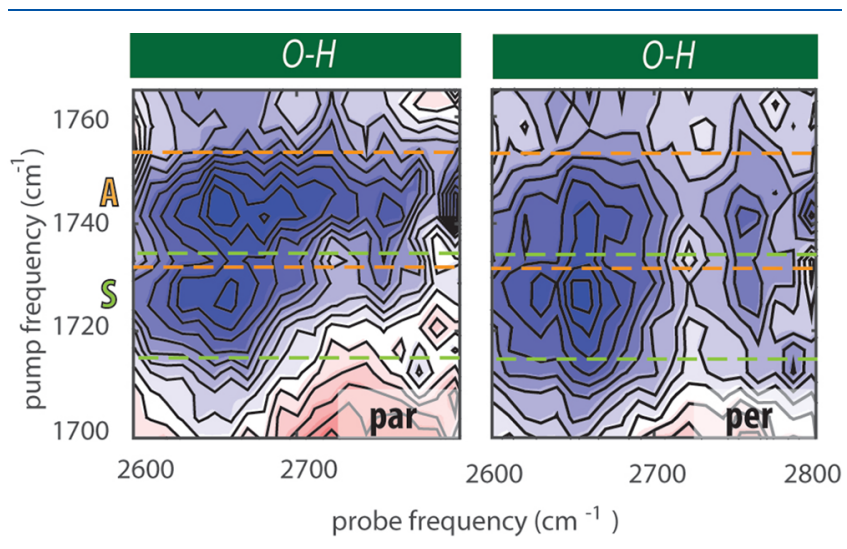

Figure 3. Cross-peak 2DIR spectra of $\mathrm{N}$-acetyl aspartic acid amide in aqueous solution in the $\mathrm{C}=\mathrm{O} / \mathrm{O}-\mathrm{H}$ cross-peak region measured in perpendicular and parallel polarization configuration at $T_{\mathrm{w}}=0.3 \mathrm{ps}$. The green and the orange bars highlight the responses of the syn and anti conformers, respectively.

dissolved in water, obtained by exciting the carbonyl vibration at $\sim 1730 \mathrm{~cm}^{-1}$ and probing the $\mathrm{O}-\mathrm{H}$ stretching vibration at $\sim 2700 \mathrm{~cm}^{-1}$. For this solution, a strong absorption band of the solvent, that is, the water $\mathrm{HOH}$-bending vibration, overlaps with the carbonyl band of the acid. Therefore, to increase the contrast between the carbonyl and the water absorption and to obtain a significant carbonyl-hydroxyl cross-peak signal, we used a $1 \mathrm{M}$ aqueous solution of $\mathrm{N}$-acetyl aspartic acid amide. The cross-peak signals that we observe in parallel and perpendicular polarization configurations for this solution are qualitatively the same as those measured for $\mathrm{N}$-acetyl aspartic acid amide dissolved in DMSO. Again we observe a stronger cross-peak signal in perpendicular polarization at a low excitation frequency of $1725 \mathrm{~cm}^{-1}$ of the carbonyl vibration. At this excitation frequency, we extract an anisotropy of approximately -0.1 , from which we derive an angle between the $\mathrm{O}-\mathrm{H}$ and $\mathrm{C}=\mathrm{O}$ groups of $\sim 65^{\circ}$. In parallel polarization, we observe a stronger cross-peak signal at a relatively high frequency of the carbonyl vibration. We find that in parallel polarization the maximum of the cross-peak intensity is at 1745 $\mathrm{cm}^{-1}$. At this excitation frequency, we extract an anisotropy of $\sim 0.25$, corresponding to an angle between $\mathrm{O}-\mathrm{H}$ and carbonyl of $\sim 30^{\circ}$.

In order to quantify the populations of the anti and syn conformers, we also measured degenerate 2DIR spectra, in which the carbonyl vibrations are both excited and probed. The 2DIR signal is proportional to the concentration and the square of the vibrational cross-section, whereas the linear IR absorption scales with the concentration and the vibrational cross-section. Hence, from the combination of the degenerate 2DIR spectrum and the linear IR absorption spectrum we can determine the ratio of the vibrational cross sections and the relative concentrations of the two carboxylic acid conformers. Figure $4 \mathrm{a}$ shows the 2DIR spectrum of $\mathrm{N}$-acetyl aspartic acid amide in aqueous solution at a concentration of $1 \mathrm{M}$. Because of the low cross-section of the water bending vibration and its a)
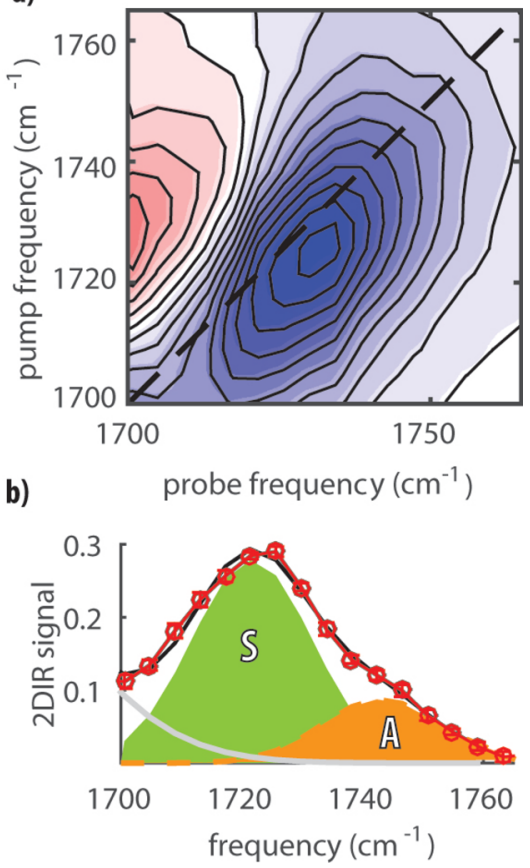

Figure 4. (a) Isotropic degenerate 2DIR spectrum of a solution of 1 $\mathrm{M} \mathrm{N}$-acetyl aspartic acid amide in water, measured at $T_{\mathrm{w}}=0.3 \mathrm{ps}$. (b) Transient absorption signal obtained by taking the diagonal slice of the bleach signal of the 2DIR spectrum plotted as a function of pump frequency. The green and orange colored bands represent the 2DIR signals of the syn and anti conformers obtained from a fit of the measured spectrum with Voigt profiles.

fast relaxation dynamics, its impact to the 2DIR signal at delay time $0.3 \mathrm{ps}$ is negligible. ${ }^{28}$ As we can observe, the diagonal peak is centered at $1725 \mathrm{~cm}^{-1}$ and is elongated at the high frequency side.

In Figure $4 b$, we plot a diagonal cut (probe = pump frequency) of the degenerate 2DIR spectrum as a function of the pump frequency. The resulting transient absorption spectrum shows a peak centered at $1725 \mathrm{~cm}^{-1}$ and a shoulder around $1745 \mathrm{~cm}^{-1}$. These two vibrational frequencies match with the excitation frequencies found in Figure 3 for the syn and anti conformers. We fit the 2DIR signal with two Voigt profiles, representing the carbonyl stretching vibrations of the syn and anti conformers. A third Voigt-shaped band centered at $1680 \mathrm{~cm}^{-1}$ was added to account for the contribution of the amide vibration (gray line). We applied the same fitting procedure to the diagonal negative absorption change of the degenerate 2DIR spectrum and linear infrared spectrum of $N$ acetyl aspartic acid amide in DMSO and found similar areas for the absorption bands of the syn and anti conformers (Figure S4). This finding implies that the vibrational cross sections of syn and anti carbonyls are similar (as it was found before ${ }^{14}$ ). It thus follows that the areas of the bands directly represent the relative abundances of the two conformers. We thus find the populations of the syn and anti conformers to be $75 \pm 10 \%$ and $25 \pm 10 \%$, respectively. These populations are similar to those found for formic acid in water. ${ }^{14}$ Hence, we conclude that the relative abundances of syn and anti conformers of carboxyl groups in polar solvents such as DMSO or water depend only weakly on the rest of the molecule.

We do not observe any cross-peak signals between the two carbonyl vibrations (exciting the anti carbonyl and probing the 
syn carbonyl or vice versa, Figure S5). This observation indicates that within the time frame of our experiment ( $\sim 5 \mathrm{ps})$ the conformers do not exchange, which is in line with a theoretical study that predicts the rotation barrier for conformational exchange of carboxyl groups to have a rather high value of $\sim 11 \mathrm{kcal} / \mathrm{mol}$ in aqueous solution. ${ }^{29}$

We performed molecular dynamics (MD) simulations to study the intermolecular interactions of the syn and anti conformers of $\mathrm{N}$-acetyl aspartic acid amide in water. The MD simulations are done with full atomistic resolution and at the force field level of theory. We ran two MD simulations of aqueous solvated $\mathrm{N}$-acetyl aspartic acid amide in the two different planar configurations of the $-\mathrm{COOH}$ group, syn and anti. The angles between $\mathrm{C}-\mathrm{O}$ and $\mathrm{O}-\mathrm{H}$ are not restrained. Further details on the system preparation and parameters can be found in the Methods section.

From the simulations of both conformers, we obtained density histograms of the hydrogen and oxygen atoms of water molecules closest to the hydrogen and oxygen atoms of the carboxyl group as a function of the hydrogen bond distance and the angle between the hydrogen bond and the corresponding covalent $\mathrm{O}-\mathrm{H}$ bond (Figure S6). We see that in all cases for distances larger than $2.45 \AA$ the angle becomes ill-defined. Thus, we define $2.45 \AA$ as a cutoff distance for hydrogen-bond formation between the carboxyl group and the water molecules.

The radial distribution functions of water oxygens forming a hydrogen bond with the acid hydrogen of the two conformers (Figure 5a) show clear differences. We find that the strongly polar $\mathrm{OH}$ group of the carboxylic acid donates $\sim 1.0$ hydrogen bond for both conformers. However, the density of water oxygen atoms at a short distance $(\sim 1.8 \AA)$ is about $10 \%$ higher for the anti conformer than for the syn conformer. This result shows that the hydrogen bonds donated by the carboxyl group of the anti conformer are shorter and stronger than those of the syn conformer. This finding reflects that the hydroxyl group is more polar for the anti conformer than for the syn conformer, in accordance with earlier theoretical work. ${ }^{26,29}$

The hydration number of the oxygen atoms is rather different for the two carboxyl group conformers (Figure $5 b, c$ ). The oxygen atom of the carbonyl group accepts $\sim 1.7$ hydrogen bonds from water molecules for the anti conformer and only $\sim 1.4$ hydrogen bonds for the syn conformer. The same trend is observed for the hydroxyl group: its oxygen atom accepts $\sim 0.7$ hydrogen bond from water molecules for the anti conformer and $\sim 0.5$ hydrogen bond for the syn conformer. The anti conformer is thus much better hydrated than the syn conformer. These differences are captured at the classical level of MD simulations, which means that they are due to exposure and steric effects and not the result of differences in the basicities of the oxygen atoms in the syn and anti conformers. In addition, we found that the average dipole moment of the anti conformer $(6.4 \mathrm{D})$ is larger than that of the syn conformer $(5.0 \mathrm{D})$. This implies that the carboxyl group in anti conformation will have stronger dipole-dipole interactions with surrounding water molecules than the carboxyl group in syn conformation. This effect will enhance the stability and propensity of the anti conformer in aqueous solutions, as has been observed for other molecules. ${ }^{30}$

The better hydration and stronger dipole-dipole interactions of the anti conformer explains why we observe this conformer to be significantly present in aqueous solution while it is nearly absent in the gas phase. The $25 \pm 10 \%$ fraction of
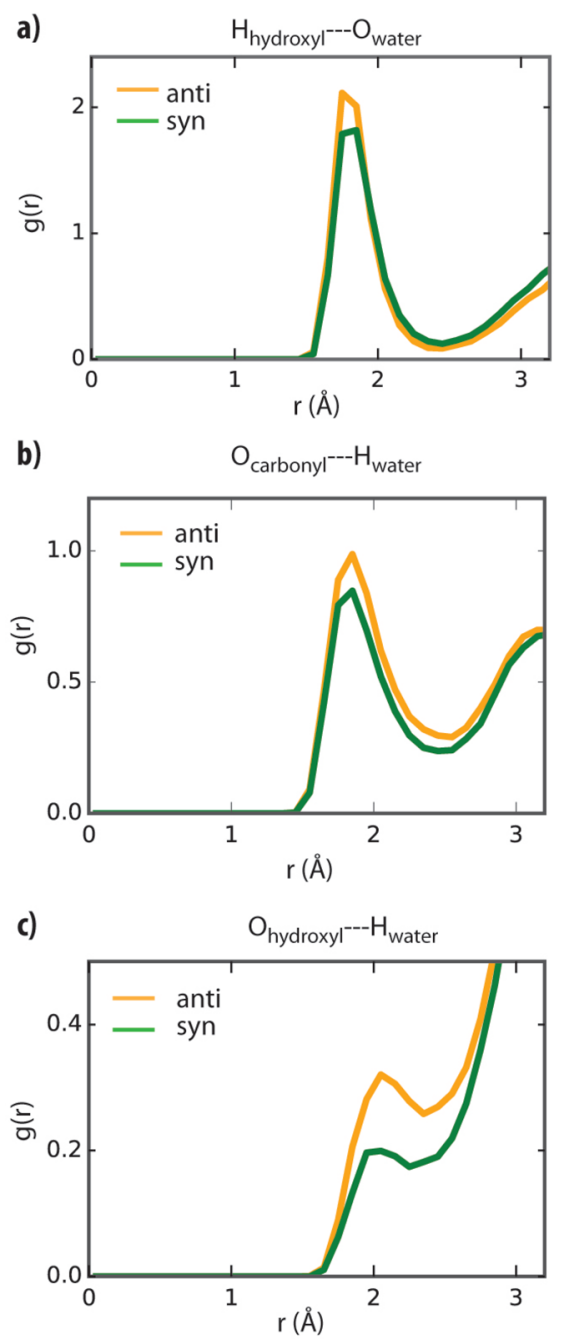

Figure 5. Molecular dynamics simulations of the interaction of the carboxyl group of $\mathrm{N}$-acetyl aspartic acid amide in syn and anti conformation with water. (a) Radial density function of the water oxygens closest to the hydrogen atom of the carboxyl group. $(b, c)$ Radial density functions of the water hydrogens closest to the carbonyl (b) and hydroxyl (c) oxygen atoms of the carboxyl group.

anti conformer in aqueous solution suggests a free energy difference between the anti and syn conformer of $0.6 \pm 0.3$ $\mathrm{kcal} / \mathrm{mol}$. This value will also determine the difference in equilibrium constants of chemical reactions involving the carboxyl group such as acid dissociation. We calculate a difference in acidity of syn and anti conformers of $\Delta \mathrm{p} K_{\mathrm{a}}=0.5$ \pm 0.3 (see Methods section). Hence, in spite of its better hydration, the anti conformer of the carboxyl group is still more acidic than the syn conformer, which can play an important role in intermolecular interactions and protolytic equilibria inside polypeptide structures. The difference in acidity between the two conformers is much smaller than the $\Delta \mathrm{p} K_{\mathrm{a}}=4$ that was obtained in calculations of the two conformers without any solvent present. ${ }^{17,31,32}$ This latter value for $\Delta \mathrm{p} K_{\mathrm{a}}$ is widely accepted in enzyme studies. ${ }^{18,20,22,33}$ The energy gap we estimate from our experiment is in fact even smaller than the $1.5-1.7 \mathrm{kcal} / \mathrm{mol}$ that has been calculated for acetic acid conformers in aqueous solution. $^{29,34,35}$ Hence, the anti conformer of the side chain carboxyl group of $\mathrm{N}$-acetyl aspartic acid amide is more acidic than the syn conformer, but this difference in acidity could 
strongly depend on the degree of solvation. Variation of the local environment of the amino acid, that is, within a protein, can thus lead to a strong change of the acidity of the aspartic acid side group.

In summary, we have shown with polarization-resolved twodimensional infrared spectroscopy that the side chain carboxyl group of $\mathrm{N}$-acetyl aspartic acid amide exists in two distinctly different conformations, both in DMSO solution and in aqueous solution. In one of these conformations, the carbonyl stretch vibration has a relatively low frequency of $\sim 1720 \mathrm{~cm}^{-1}$, and the angle between the carbonyl and hydroxyl groups of the carboxylic acid side group amounts to $\sim 90^{\circ}$. For the other conformer, the carbonyl stretch vibration has a relatively high frequency of $\sim 1745 \mathrm{~cm}^{-1}$, and the angle between the carbonyl and hydroxyl groups of the carboxylic acid side group is $\sim 35^{\circ}$. In line with earlier work, we assign these conformers to nearly planar syn and anti conformers, where we explain the deviations from the ideal values of $60^{\circ}$ (syn) and $0^{\circ}$ (anti) from small angle out-of-plane rotations of the hydroxyl and carbonyl groups.

We find that the anti conformer of $N$-acetyl aspartic acid amide has a relative abundance of $\sim 25 \%$, when dissolved both in DMSO and in water, which is significantly higher than the $<1 \%$ observed in gas-phase studies. Molecular dynamics simulations show that the increase of the relative abundance of the anti conformer upon dissolution in water can be explained by the more favorable hydration of this conformer in comparison to the syn conformer. The lower relative abundance of the anti conformer in comparison to the syn conformer implies that this conformer is more acidic in water than the syn conformer, corresponding to a $\Delta \mathrm{p} K_{\mathrm{a}}$ of $0.5 \pm 0.3$. The acid dissociation is only one example of the chemical properties of the carboxyl group that depend on the molecular conformation. Hence, the fact that both the syn and the anti conformers are present under biological conditions must be carefully taken into account when studying biochemical processes of polypeptides.

\section{METHODS}

Sample Preparation. $N$-Acetyl aspartic acid amide (>95\%, Enamine Ltd.) was dissolved in either deuterated dimethyl sulfoxide (DMSO- $d_{6}$, anhydrous, $99.8 \%$ D, Sigma-Aldrich) or in ultrapure water to reach the desired concentration. For the infrared absorption measurements (Bruker Vertex 80v FTIR spectrometer) and the two-dimensional infrared experiments, the solution was held between two calcium fluoride windows separated by a PTFE spacer of $10-50 \mu \mathrm{m}$ thickness.

2DIR Experiments. We excite the carbonyl $(\mathrm{C}=\mathrm{O})$ vibration with an intense femtosecond mid-infrared pulse, and we probe the vibrational response of the hydroxyl $(\mathrm{O}-\mathrm{H})$ vibration with a delayed weak mid-infrared pulse. The excitation of the carbonyl vibration will affect the response of the hydroxyl vibration of the same carboxyl group resulting in a transient absorption change that is denoted as a crosspeak. Due to the extremely broad response of the acidic $\mathrm{OH}$ groups, an additional isotropic background signal appears, which we account for with a sloping line. By resolving the cross-peak along the excitation frequency, we identify different conformers of the carboxyl group. By measuring the cross-peak signal in both parallel and perpendicular polarization configuration of the excitation and probing pulses, we determine the angle between the transition dipole moments of the hydroxyl and carbonyl vibrations (indicated with blue and red arrows in Figure 1). Additionally, degenerate 2DIR experiments exciting and probing carbonyl vibrations are performed to determine the relative populations of the different conformers. ${ }^{14}$ The 2DIR signal is proportional to $\sigma^{2} C$, where $\sigma$ is the vibrational cross-section and $C$ is the concentration, while the linear absorption signal is proportional to $\sigma C$. From the comparison of the linear absorption and the 2D-IR spectra, we can determine the values of the concentration $C$ and cross-section $\sigma$ of the syn and anti conformers and thereby determine their molar ratio, $x_{\text {syn }} / x_{\text {anti. }}$. We calculate the free energy difference, $\Delta G$, for the two conformers as $\Delta G=k_{\mathrm{B}} T \ln \left(x_{\text {syn }} / x_{\text {anti }}\right)$. Using $\Delta G$, we determine the difference in acidity equilibrium constant: $\Delta \mathrm{p} K_{\mathrm{a}}=\Delta G /\left(k_{\mathrm{B}} T \ln 10\right)$.

The details of our 2DIR setup can be found in the literature. ${ }^{36}$ In brief, we produced the pump and probe pulse by two independently tunable optical parametric amplifiers pumped by the $800 \mathrm{~nm}$ output of a Ti:sapphire regenerative amplifier. A Mach-Zehnder interferometer was used to generate the pump pulse pair $(\sim 100 \mathrm{fs}, 5 \mu \mathrm{J}$ per pulse), which was subsequently focused in the sample to excite the carbonyl vibrations. This excitation induces transient absorption changes that are detected with a weak $(0.35 \mu \mathrm{J})$ single femtosecond probe pulse that is overlapped with the pump pulse in the sample and delayed by a time $T_{\mathrm{w}}$. In all experiments, the excitation pulses are centered at $1730 \mathrm{~cm}^{-1}$ with a bandwidth of $200 \mathrm{~cm}^{-1}$, in resonance with the carbonyl vibrations. The probe pulse was centered at $2650 \mathrm{~cm}^{-1}$ to measure the response of the $\mathrm{O}-\mathrm{H}$ stretch vibrations in dimethyl sulfoxide and water or at $1730 \mathrm{~cm}^{-1}$ to measure the response of $\mathrm{C}=\mathrm{O}$ stretch vibrations. The polarization of probe pulse was rotated at $45^{\circ}$ with respect to the pump by a halfwave plate. After the sample the probe was split, and the parallel and perpendicular polarization components were dispersed by a monochromator and measured simultaneously by two arrays of a mercury-cadmium-telluride (MCT) detector; the third array of the detector was used to measure the reference pulse to account for pulse-to-pulse fluctuations. The signal at each pixel was measured while scanning the delay time between the two pump pulses and then Fourier transformed to obtain dependence of the 2DIR signal on the pump frequency. As a result, we obtain the 2DIR transient absorption signal plotted as a function of pump and probe frequencies detected in parallel and perpendicular polarization configurations.

Molecular Dynamics Simulations. The $N$-acetyl aspartic acid amide molecule is generated with the AmberTools 18 xleap module ${ }^{37}$ and prepared with GROMACS 5.1.4. ${ }^{38}$ It is treated with the CHARMM27 force field ${ }^{39}$ and placed in cubic box of $2.7 \times 2.7 \times 2.7 \mathrm{~nm}^{3}$ containing a density of $1000 \mathrm{~g} / \mathrm{L}$ water molecules, which are treated with the TIP3P force field. ${ }^{40}$ No counterions are added. The used parameters of $N$ acetyl aspartic acid amide (CHARMM2 $7^{39}$ ) and water $\left(\mathrm{TIP} 3 \mathrm{P}^{40}\right)$ are chosen to be software-recommended and tested for protein structure. ${ }^{41}$ We perform energy minimization followed by a $100 \mathrm{~ns}$ equilibration in an NPT (constant number of particles, pressure, and temperature) ensemble with the CSVR (canonical sampling through velocity rescaling) thermostat $^{42}$ set to $300 \mathrm{~K}$ with a time constant of $0.1 \mathrm{ps}$ and the Parrinello-Rahman barostat ${ }^{43}$ set to 1.0 bar with a time constant of $1.0 \mathrm{ps}$. The MD time step size is $2.0 \mathrm{fs}$. We then initiate two new $100 \mathrm{~ns}$ runs, one in the syn and another one in the anti conformation. The $\mathrm{O}-\mathrm{C}-\mathrm{O}-\mathrm{H}$ dihedral angle cosine 
was harmonically restrained to 1.0 (syn) or -1.0 (anti) with a force constant of $50 \mathrm{kcal} / \mathrm{mol}$. Removing this restraint did not have an effect on the radial distribution functions or hydrogenbond distances and hydrogen-bond coordination for the syn conformer, but for the anti conformer this restraint is needed to maintain its conformation during the long simulation time interval. Analysis is done with PLUMED 2.3.0 ${ }^{44}$ and VMD 1.9.4a12. ${ }^{45}$ We take the last 50 ns of these runs, with data points every 2 ps, for analysis. The number of hydrogen bonds for the carboxyl group hydrogen and the two oxygens is calculated simply by counting the number of hydrogenacceptor pairs within the cutoff distance $(2.45 \AA$ as determined from Figure S6) and averaging.

\section{ASSOCIATED CONTENT}

\section{SI Supporting Information}

The Supporting Information is available free of charge at https://pubs.acs.org/doi/10.1021/acs.jpclett.0c00711.

Infrared absorption spectra of $\mathrm{N}$-acetyl aspartic acid amide in water and DMSO, cross-peak 2DIR spectra of $\mathrm{N}$-acetyl leucine and $\mathrm{N}$-acetyl phenylalanine in DMSO, degenerate 2DIR spectrum of $N$-acetyl aspartic acid amide in DMSO, and density histograms of the water oxygen and hydrogen atoms in the vicinity of the carboxyl group of $N$-acetyl aspartic acid amide (PDF)

\section{AUTHOR INFORMATION}

\section{Corresponding Author}

Huib J. Bakker - AMOLF, 1098 XG Amsterdam, The

Netherlands; 이이.org/0000-0003-1564-5314;

Email: h.bakker@amolf.nl

\section{Authors}

Oleksandr O. Sofronov - AMOLF, 1098 XG Amsterdam, The Netherlands; 이이이.org/0000-0001-7744-6404

Giulia Giubertoni - AMOLF, 1098 XG Amsterdam, The

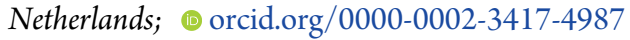

Alberto Pérez de Alba Ortíz - Amsterdam Center for Multiscale Modeling and Van 't Hoff Institute for Molecular Sciences, University of Amsterdam, 1098 XH Amsterdam, The Netherlands

Bernd Ensing - Amsterdam Center for Multiscale Modeling and Van 't Hoff Institute for Molecular Sciences, University of Amsterdam, 1098 XH Amsterdam, The Netherlands; 당 orcid.org/0000-0002-4913-3571

Complete contact information is available at: https://pubs.acs.org/10.1021/acs.jpclett.0c00711

\section{Author Contributions}

"IIO.O.S. and G.G. equally contributed.

\section{Notes}

The authors declare no competing financial interest.

\section{ACKNOWLEDGMENTS}

This work is part of the research program of the Netherlands Organization for Scientific Research (NWO) and was performed at the NWO research institute AMOLF. This work is part of the industrial partnership programme Hybrid Soft Materials that is carried out under an agreement between Unilever Research and the Netherlands Organization for Scientific Research (NWO). This project has received funding from the European Research Council (ERC) under the
European Union's Horizon 2020 research and innovation program (Grant Agreement No 694386). A. Pérez de Alba Ortiz acknowledges funding from the Mexican National Council for Science and Technology (CONACYT).

\section{REFERENCES}

(1) Mwaura, J.; Tao, Z.; James, H.; Albers, T.; Schwartz, A.; Grewer, C. Protonation state of a conserved acidic amino acid involved in $\mathrm{Na}$ + binding to the glutamate transporter EAAC1. ACS Chem. Neurosci. 2012, 3, 1073-1083.

(2) Di Nardo, G.; Breitner, M.; Bandino, A.; Ghosh, D.; Jennings, G. K.; Hackett, J. C.; Gilardi, G. Evidence for an Elevated Aspartate $p K_{a}$ in the Active Site of Human Aromatase. J. Biol. Chem. 2015, 290, $1186-1196$.

(3) Hanz, S. Z.; Shu, N. S.; Qian, J.; Christman, N.; Kranz, P.; An, M.; Grewer, C.; Qiang, W. Protonation-Driven Membrane Insertion of a pH-Low Insertion Peptide. Angew. Chem., Int. Ed. 2016, 55, $12376-12381$.

(4) Gunner, M. R.; Alexov, E. A pragmatic approach to structure based calculation of coupled proton and electron transfer in proteins. Biochim. Biophys. Acta, Bioenerg. 2000, 1458, 63-87.

(5) Davis, C. M.; Dyer, R. B. The Role of Electrostatic Interactions in Folding of $\beta$-Proteins. J. Am. Chem. Soc. 2016, 138, 1456-1464.

(6) Isom, D. G.; Castañeda, C. A.; Cannon, B. R.; Velu, P. D.; García-Moreno E., B. Charges in the hydrophobic interior of proteins. Proc. Natl. Acad. Sci. U. S. A. 2010, 107, 16096.

(7) Pedersen, B. P.; Buch-Pedersen, M. J.; Preben Morth, J.; Palmgren, M. G.; Nissen, P. Crystal structure of the plasma membrane proton pump. Nature 2007, 450, 1111-1114.

(8) Garczarek, F.; Gerwert, K. Functional waters in intraprotein proton transfer monitored by FTIR difference spectroscopy. Nature 2006, 439, 109-112.

(9) Jasti, J.; Furukawa, H.; Gonzales, E. B.; Gouaux, E. Structure of acid-sensing ion channel 1 at $1.9 \AA$ resolution and low pH. Nature 2007, 449, 316-323.

(10) Yoder, N.; Yoshioka, C.; Gouaux, E. Gating mechanisms of acid-sensing ion channels. Nature 2018, 555, 397-401.

(11) Pettersson, M.; Maçôas, E. M.; Khriachtchev, L.; Fausto, R.; Räsänen, M. Conformational isomerization of formic acid by vibrational excitation at energies below the torsional barrier. J. Am. Chem. Soc. 2003, 125, 4058-4059.

(12) Pettersson, M.; Lundell, J.; Khriachtchev, L.; Rasanen, M. IR spectrum of the other rotamer of formic acid, cis-HCOOH. J. Am. Chem. Soc. 1997, 119, 11715-11716.

(13) Maçôas, E. M. S.; Khriachtchev, L.; Pettersson, M.; Fausto, R.; Räsänen, M. Rotational isomerization of small carboxylic acids isolated in argon matrices: Tunnelling and quantum yields for the photoinduced processes. Phys. Chem. Chem. Phys. 2005, 7, 743-749.

(14) Giubertoni, G.; Sofronov, O. O.; Bakker, H. J. Observation of Distinct Carboxylic Acid Conformers in Aqueous Solution. J. Phys. Chem. Lett. 2019, 10, 3217-3222.

(15) Maçôas, E. M. S.; Khriachtchev, L.; Pettersson, M.; Fausto, R.; Räsänen, M. Internal Rotation in Propionic Acid: Near-InfraredInduced Isomerization in Solid Argon. J. Phys. Chem. A 2005, 109, $3617-3625$.

(16) D’Ascenzo, L.; Auffinger, P. A comprehensive classification and nomenclature of carboxyl-carboxyl(ate) supramolecular motifs and related catemers: implications for biomolecular systems. Acta Crystallogr., Sect. B: Struct. Sci., Cryst. Eng. Mater. 2015, 71, 164-175.

(17) Gandour, R. D. On the importance of orientation in general base catalysis by carboxylate. Bioorg. Chem. 1981, 10, 169-176.

(18) Salmon, M.; Thimmappa, R. B.; Minto, R. E.; Melton, R. E.; Hughes, R. K.; O'maille, P. E.; Hemmings, A. M.; Osbourn, A. A conserved amino acid residue critical for product and substrate specificity in plant triterpene synthases. Proc. Natl. Acad. Sci. U. S. A. 2016, 113, E4407-E4414. 
(19) Świderek, K.; Tuñón, I.; Moliner, V.; Bertran, J. Protein Flexibility and Preorganization in the Design of Enzymes. The Kemp Elimination Catalyzed by HG3.17. ACS Catal. 2015, 5, 2587-2595.

(20) Bahnson, B. J.; Anderson, V. E.; Petsko, G. A. Structural mechanism of enoyl-CoA hydratase: Three atoms from a single water are added in either an E1cb stepwise or concerted fashion. Biochemistry 2002, 41, 2621-2629.

(21) Debler, E. W.; Ito, S.; Seebeck, F. P.; Heine, A.; Hilvert, D.; Wilson, I. A. Structural origins of efficient proton abstraction from carbon by a catalytic antibody. Proc. Natl. Acad. Sci. U. S. A. 2005, 102, 4984-4989.

(22) Eriksson, A.; Kürten, C.; Syrén, P.-O. Protonation-Initiated Cyclization by a Class II Terpene Cyclase Assisted by Tunneling. ChemBioChem 2017, 18, 2301-2305.

(23) Khavrutskii, I. V.; Compton, J. R.; Jurkouich, K. M.; Legler, P. M. Paired Carboxylic Acids in Enzymes and Their Role in Selective Substrate Binding, Catalysis, and Unusually Shifted $\mathrm{p} \mathrm{K}$ a Values. Biochemistry 2019, 58, 5351-5365.

(24) Gündoğdu, K.; Bandaria, J.; Nydegger, M.; Rock, W.; Cheatum, C. M. Relaxation and anharmonic couplings of the $\mathrm{O}-\mathrm{H}$ stretching vibration of asymmetric strongly hydrogen-bonded complexes. J. Chem. Phys. 2007, 127, 044501.

(25) Stingel, A. M.; Petersen, P. B. Couplings Across the Vibrational Spectrum Caused by Strong Hydrogen Bonds: A Continuum 2D IR Study of the 7-Azaindole-Acetic Acid Heterodimer. J. Phys. Chem. B 2016, 120, 10768-10779.

(26) Pal, R.; Reddy, M. B. M.; Dinesh, B.; Venkatesha, M. A.; Grabowsky, S.; Jelsch, C.; Guru Row, T. N. Syn vs Anti Carboxylic Acids in Hybrid Peptides: Experimental and Theoretical Charge Density and Chemical Bonding Analysis. J. Phys. Chem. A 2018, 122, 3665-3679.

(27) Moilanen, D. E.; Fenn, E. E.; Lin, Y.-S.; Skinner, J. L.; Bagchi, B.; Fayer, M. D. Water inertial reorientation: Hydrogen bond strength and the angular potential. Proc. Natl. Acad. Sci. U. S. A. 2008, 105, $5295-5300$.

(28) Hume, S.; Hithell, G.; Greetham, G. M.; Donaldson, P. M.; Towrie, M.; Parker, A. W.; Baker, M. J.; Hunt, N. T. Measuring proteins in $\mathrm{H} 2 \mathrm{O}$ with 2D-IR spectroscopy. Chemical Science 2019, 10, 6448-6456.

(29) Nagy, P. I. The syn-anti equilibrium for the $\mathrm{COOH}$ group reinvestigated. Theoretical conformation analysis for acetic acid in the gas phase and in solution. Comput. Theor. Chem. 2013, 1022, 59-69.

(30) Cezar, H. M.; Canuto, S.; Coutinho, K. Solvent effect on the syn/anti conformational stability: A comparison between conformational bias Monte Carlo and molecular dynamics methods. Int. J. Quantum Chem. 2019, 119, No. e25688.

(31) Peterson, M. R.; Csizmadia, I. G. Determination and analysis of the formic acid conformational hypersurface. J. Am. Chem. Soc. 1979, 101, 1076-1079.

(32) Wiberg, K. B.; Laidig, K. E. Barriers to rotation adjacent to double bonds. 3. The carbon-oxygen barrier in formic acid, methyl formate, acetic acid, and methyl acetate. The origin of ester and amide resonance. J. Am. Chem. Soc. 1987, 109, 5935-5943.

(33) Hammer, S. C.; Marjanovic, A.; Dominicus, J. M.; Nestl, B. M.; Hauer, B. Squalene hopene cyclases are protonases for stereoselective Brønsted acid catalysis. Nat. Chem. Biol. 2015, 11, 121-126.

(34) Pranata, J. Relative basicities of carboxylate lone pairs in aqueous solution. J. Comput. Chem. 1993, 14, 685-690.

(35) Sato, H.; Hirata, F. The syn-/anti-conformational equilibrium of acetic acid in water studied by the RISM-SCF/MCSCF method. J. Mol. Struct.: THEOCHEM 1999, 461-462, 113-120.

(36) Selig, O.; Siffels, R.; Rezus, Y. L. A. Ultrasensitive Ultrafast Vibrational Spectroscopy Employing the Near Field of Gold Nanoantennas. Phys. Rev. Lett. 2015, 114, 233004.

(37) Case, D.; Ben-Shalom, I.; Brozell, S.; Cerutti, D.; Cheatham, T., III; Cruzeiro, V.; Darden, T.; Duke, R.; Ghoreishi, D.; Gilson, M., et al. AMBER 2018; University of California, San Francisco, 2018.
(38) Berendsen, H. J. C.; van der Spoel, D.; van Drunen, R. GROMACS: a message-passing parallel molecular dynamics implementation. Comput. Phys. Commun. 1995, 91, 43-56.

(39) Feller, S. E.; MacKerell, A. D. An improved empirical potential energy function for molecular simulations of phospholipids. J. Phys. Chem. B 2000, 104, 7510-7515.

(40) Jorgensen, W. L.; Chandrasekhar, J.; Madura, J. D.; Impey, R. W.; Klein, M. L. Comparison of simple potential functions for simulating liquid water. J. Chem. Phys. 1983, 79, 926-935.

(41) Bjelkmar, P.; Larsson, P.; Cuendet, M. A.; Hess, B.; Lindahl, E. Implementation of the CHARMM force field in GROMACS: analysis of protein stability effects from correction maps, virtual interaction sites, and water models. J. Chem. Theory Comput. 2010, 6, 459-466.

(42) Bussi, G.; Donadio, D.; Parrinello, M. Canonical sampling through velocity rescaling. J. Chem. Phys. 2007, 126, 014101.

(43) Parrinello, M.; Rahman, A. Polymorphic transitions in single crystals: A new molecular dynamics method. J. Appl. Phys. 1981, 52, $7182-7190$.

(44) Tribello, G. A.; Bonomi, M.; Branduardi, D.; Camilloni, C.; Bussi, G. PLUMED 2: New feathers for an old bird. Comput. Phys. Commun. 2014, 185, 604-613.

(45) Humphrey, W.; Dalke, A.; Schulten, K. VMD - Visual Molecular Dynamics. J. Mol. Graphics 1996, 14, 33-38. 